\title{
Metallicities of intermediate-age and old star clusters in the Large Magellanic Cloud
}

\author{
Arūnas Kučinskas, ${ }^{1,2}$ Vidas Dobrovolskas, ${ }^{2}$ Algimantas Černiauskas, ${ }^{3,1}$ \\ Roma Lazauskaite் $\dot{e}^{3}$ and Toshihiko Tanabé ${ }^{4}$ \\ ${ }^{1}$ Vilnius University, Institute of Theoretical Physics and Astronomy, Vilnius, Lithuania \\ ${ }^{2}$ Astronomical Observatory of Vilnius University, Lithuania \\ ${ }^{3}$ Department of Physics, Vilnius Pedagogical University, Lithuania \\ ${ }^{4}$ Institute of Astronomy, University of Tokyo, Japan
}

\begin{abstract}
We derive photometric metallicities for 56 intermediate-age and old star clusters in the Large Magellanic Cloud from the slopes of their red-giant branches in near-infrared colormagnitude diagrams (CMDs). The cluster sample covers the LMC bar and disk to a distance of $\sim 2 \mathrm{kpc}$ from the LMC center. The derived cluster metallicity distribution spans a range of $[\mathrm{Fe} / \mathrm{H}]=+0.0 \ldots 1.4$ and is similar to the metallicity distribution of field stars. We find that the average cluster metallicities in certain fields of the LMC bar and disk may be different, but there seems to be no compelling evidence for a global cluster metallicity gradient in the LMC.
\end{abstract}

Keywords. Magellanic Clouds, galaxies: star clusters, galaxies: abundances

The full poster (in pdf format) is available at

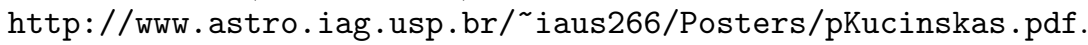

